\title{
Opioid-Mediated Modulation of Acid-Sensing Ion Channel Currents in Adult Rat Sensory Neurons
}

\author{
Malgorzata Zaremba and Victor Ruiz-Velasco \\ Ruiz-Velasco Laboratory, Department of Anesthesiology and Perioperative Medicine, Penn State College of Medicine, \\ Hershey, Pennsylvania
}

Received October 16, 2018; accepted February 20, 2019

\section{ABSTRACT}

Muscle ischemia, associated with peripheral artery disease (PAD), leads to the release of proinflammatory mediators that decrease extracellular $\mathrm{pH}$ and trigger the activation of protonactivated acid-sensing ion channels (ASIC). Claudication pain, linked with low blood flow, can be partially relieved by endogenous opioid peptide release. However, we previously reported that sustained ASIC currents in dorsal root ganglion (DRG) neurons were enhanced by naturally occurring endomorphin-1 and -2 opioid peptides, indicating a role of opioid involvement in hyperalgesia. The present study examined whether clinically employed synthetic (fentanyl, remifentanil) and the semisynthetic opioid (oxycodone) would also potentiate sustained ASIC currents, which arise from ASIC3 channel isoforms. Here, we show that exposure of each opioid to DRG neurons resulted in potentiation of the sustained ASIC currents. On the other hand, the potentiation was not observed in DRG neurons from ASIC3 knockout rats. Further, the enhancement of the ASIC currents was resistant to pertussis toxin treatment, suggesting that $\mathrm{G} \alpha_{\mathrm{i}} / \mathrm{G} \alpha_{\mathrm{o}}$ G-proteins are not involved. Additionally, the potentiation of sustained ASIC currents was greater in DRG neurons isolated from rats with ligated femoral arteries (a model of PAD). The effect of all three opioids on the transient ASIC peak current was mixed (increase, decrease, no effect). The inhibitory action appears to be mediated by the presence of ASIC1 isoform, while the potentiating effect is primarily due to ASIC3 isoform expression. These findings reveal that, under certain conditions, these three opioids can increase ASIC channel activity, possibly giving rise to opioid-induced hyperalgesia.

\section{Introduction}

Acid-sensing ion channels (ASIC) belong to the epithelial $\mathrm{Na}^{+}$channel/degenerin (ENaC/DEG) family and are primarily $\mathrm{Na}^{+}$-selective channels that open in response to a drop in extracellular $\mathrm{pH}$. In mammals, there are six ASIC isoforms (ASIC1a and 1b, ASIC2a and 2b, ASIC3, and ASIC4) produced by four genes (Baron and Lingueglia, 2015; Kellenberger and Schild, 2015). A functional ASIC channel is made up of three subunits and is either homomeric or heteromeric (Kellenberger and Schild, 2015). ASIC1-3 are expressed primarily in central and peripheral nervous systems (Deval and Lingueglia, 2015). In the peripheral nervous system, ASIC1 and ASIC3 are found in trigeminal and dorsal root ganglion (DRG) sensory neurons (Deval and Lingueglia, 2015).

Although DRG neurons express primarily ASIC1 and ASIC3 isoforms, their stoichiometry is not known. Moreover, under ischemic conditions the expression levels of both isoforms have been reported to be altered (Liu et al., 2010; Walder et al., 2010; Farrag et al., 2017). Both variants exhibit a high degree of

This work was supported by grants from the National Institutes of Health National Institute of Arthritis and Musculoskeletal and Skin Diseases (NIAMS) [R01 AR-059397] and National Heart, Lung, and Blood Institute (NHLBI) [P01 HL-130987].

https://doi.org/10.1124/mol.118.114918.
$\mathrm{H}^{+}$ion sensitivity, and their threshold for activation occurs when the extracellular $\mathrm{pH}$ reaches 7.2 (Gründer and Pusch, 2015; Kellenberger and Schild, 2015). ASIC3 channel stimulation results in a biphasic current that is unique to this subunit. There is an initial rapidly inactivating current that is followed by a sustained current that lasts as long as the external environment remains acidic (Gründer and Pusch, 2015). The inactivation time constants $(\tau)$ for ASIC1a and ASIC3 are 1.23.4 and 0.3 seconds, respectively (Kellenberger and Schild, 2015). The difference in this biophysical parameter provides a convenient way to distinguish ASIC1 from ASIC3.

Recent studies have shown modulation of ASIC currents by signaling molecules that are released during chronic inflammatory conditions. For instance, lactic acid can enhance ASIC1 and ASIC3 peak current in sensory neurons (Immke and McCleskey, 2001; Molliver et al., 2005). Enhancement of sustained ASIC3 currents by arachidonic acid and lysophosphatidylcholine has been described elsewhere (Smith et al., 2007; Marra et al., 2016). Likewise, the endogenous opioid peptides, dynorphins, which stimulate kappa opioid receptors, have been reported to potentiate ASIC1 currents independent of G protein coupling (Sherwood and Askwith, 2009).

In addition, limb ischemia in people with peripheral artery disease (PAD) can lead to intermittent claudication-leg pain

ABBREVIATIONS: ASIC, acid-sensing ion channels; DAMGO, [D-Ala ${ }^{2}, \mathrm{~N}-\mathrm{MePhe}^{4}, \mathrm{Gly}$-ol]-enkephalin; Dil, DilC ${ }_{12}(3)$-tetramethylindocarbocyanine perchlorate; DRG, dorsal root ganglion; E-1/2, endomorphin 1/2; EGFP, enhanced green fluorescent protein; Fen, fentanyl; FP, freely profused; KO, knockout; LIG, ligated; MOR, mu opioid receptor; OIH, opioid-induced hyperalgesia; Oxy, oxycodone; PAD, peripheral artery disease; PTX, pertussis toxin; Rem, remifentanil; $\tau$, inactivation time constant; WKY, Wistar-Kyoto. 
induced by walking (Criqui and Aboyans, 2015). The pain associated with claudication partly results from tissue acidosis and inflammatory mediators, and is transduced by thin muscle afferents. Prescription opioids make up one of the treatment modalities for PAD patients with claudication, but their use is limited by their tendency to cause tolerance, physical dependence, and addiction resulting from the loss of MOR at the surface level.

Moreover, the use of opioids for relief of either chronic pain or postsurgical pain can, under certain conditions, lead to paradoxical pain known as opioid-induced hyperalgesia (OIH). OIH, a nociceptive sensitization state, occurs when the administration of opioids intended for pain relief leads to an increased sensitivity to painful stimuli (Lee et al., 2011).

Endogenous opioid peptides are known to be released at ischemic or inflammatory sites (Mousa et al., 2002). Previously, we found that the high-affinity mu opioid receptor (MOR) peptides, endomorphin 1 (E-1) and endomorphin 2 (E-2), potentiated sustained ASIC3 currents in the absence of MOR stimulation and G protein signaling (Farrag et al., 2017). Thus, the present study expands on these findings and determines whether the clinically employed opioids fentanyl
(Fen), remifentanil (Rem), and oxycodone (Oxy) would also potentiate ASIC3 currents. The synthetic opioids Fen and Rem (see Fig. 1A) have been reported to induce $\mathrm{OIH}$ when administered after surgery (Fletcher and Martinez, 2014, and references therein). Whether administration of the semisynthetic opioid Oxy (Fig. 1A) can lead to OIH is unknown.

\section{Materials and Methods}

Animals. All experiments were approved by the Penn State College of Medicine or Medical College of Wisconsin Institutional Animal Care and Use Committee (IACUC) and complied with the National Institutes of Health guidelines. DRG neurons were isolated from adult male Sprague-Dawley rats (125-175 g; Charles River, King of Prussia, PA). For experiments shown in Fig. 3, Wistar-Kyoto (WKY) and transgenic WKY ASIC3 knockout (KO) $\left(\mathrm{ASIC}^{--}\right)$rats were employed. Wild-type and mutant strain WKY rats were obtained from the Gene Editing Rat Resource Center (Melinda Dwinell, Ph.D., PI/NHLBI; Medical College of Wisconsin). This ASIC3 KO strain

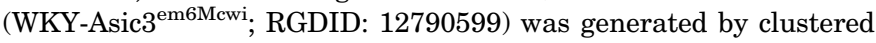
regularly interspaced short palindromic repeats (CRISPR)/Cas9 technology, targeting the sequence GGCCCACAGCCCTCGGCGCAGGG (protospacer adjacent motif underlined) into WKY/NCrl (Charles

\section{A Opioid Chemical Structures}

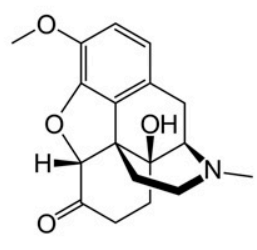

Oxycodone

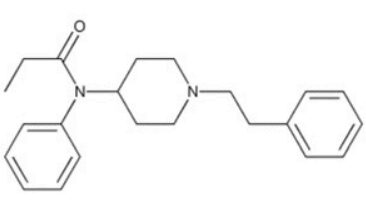

Fentanyl

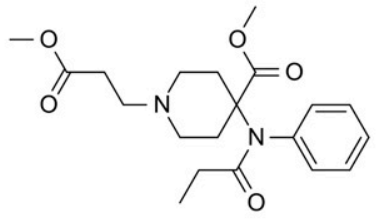

Remifentanil

\section{B ASIC current recording protocol}

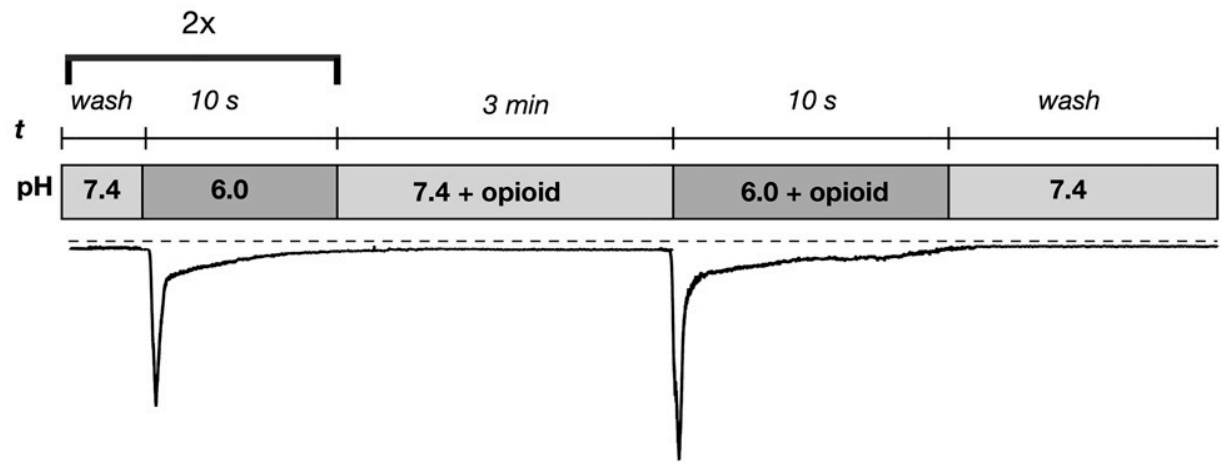

\section{Data analysis}

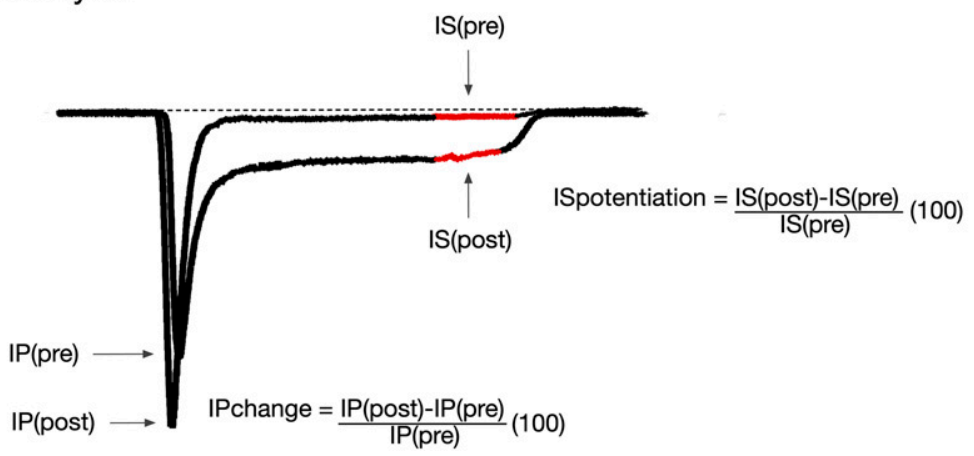

Fig. 1. ASIC current activation protocol and analysis. (A) Opioid chemical structures. (B) Protocol for ASIC current recording. Cells were exposed to control solution $(\mathrm{pH} 6.0)$ twice to verify stability of current. Thereafter, the cells were preincubated for 3 minutes in external solution containing opioid ( $\mathrm{pH}$ 7.4). Afterward, the external solution was switched to $\mathrm{pH} 6.0+$ opioid for 10 seconds, followed by a return to a solution of $\mathrm{pH}$ 7.4. (C) Data analysis of the $\mathrm{pH}$-mediated potentiation of the sustained ASIC currents $\left(\mathrm{I}_{\mathrm{S}}\right)$, where $\mathrm{I}_{\mathrm{S}}($ pre) is the sustained current amplitude in the control solution ( $\mathrm{pH} \mathrm{6.0)}$ and $\mathrm{I}_{\mathrm{S}}$ (post) is the sustained current amplitude in the test solution $(\mathrm{pH} 6.0+$ opioid $)$. The current amplitude was measured as the average current amplitude obtained during the last second of the "test" pulse (shown in red). The transient peak ASIC current $\left(\mathrm{I}_{\mathrm{P}}\right)$ amplitude was measured at the peak of the current obtained at $\mathrm{pH} 6.0, \mathrm{I}_{\mathrm{P}}(\mathrm{pre})$, and at $\mathrm{pH} 6.0+$ opioid, $\mathrm{I}_{\mathrm{P}}$ (post). 
River Laboratories) rat embryos, resulting in 61 base pair deletions in exon 1 and predicted truncation of the normal ASIC3 open reading frame after only 22 amino acids.

Femoral Artery Ligation and DRG Neuron Labeling. Three days before the DRG neuron isolation, bilateral femoral artery ligations were performed with 5 -O silk sutures under anesthesia (3\%-5\% isoflurane), as described elsewhere (Copp et al., 2016). The wounds were closed with stainless steel wound clips. Under these conditions, there is a reduction of blood flow reserve capacity that nevertheless is sufficient to meet the metabolic demands at rest (Lash et al., 1995). Additionally, the triceps surae muscles were injected with the fluorescent neuronal tracer $3 \% \mathrm{DilC}_{12}(3)$-tetramethylindocarbocyanine perchlorate (Dil; Thermo Fisher Scientific, Carlsbad, CA) prepared in dimethylsulfoxide. A total of $30 \mu \mathrm{l}$ of Dil was injected per leg with a 30 -gauge needle, which was left in the muscle for approximately 10 seconds to avoid tracer outflow.

DRG Neuron Isolation and Dissociation. Three days after artery ligation and tracer injection, the rats were anesthetized with $\mathrm{CO}_{2}$ and sacrificed by decapitation. The lumbar $\left(\mathrm{L}_{4}-\mathrm{L}_{5}\right)$ DRG were dissected and rapidly placed in ice-cold Hank's balanced salt solution (Sigma-Aldrich, St. Louis, MO) and cleared of connective tissue. Thereafter, the ganglia were enzymatically dissociated in Earle's balanced salt solution (Sigma-Aldrich) containing collagenase Type D (0.6 mg/ml; Roche, Mannheim, Germany), trypsin $(0.35 \mathrm{mg} / \mathrm{ml}$; Worthington, Lakewood, NJ), and DNase $(0.1 \mathrm{mg} / \mathrm{ml}$; Alfa Aesar, Ward Hill, MA) and shaken in a water bath for 40 minutes at $35^{\circ} \mathrm{C}$. Afterward, the neurons were centrifuged twice for 6 minutes at $50 \mathrm{~g}$.

Thereafter, the DRG neurons were placed in minimum essential medium (Thermo Fisher Scientific) supplemented with 10\% FBS, 1\% glutamine, and $1 \%$ penicillin-streptomycin. The dissociated neurons were then plated onto poly-L-lysine-coated $35-\mathrm{mm}$ polystyrene dishes and incubated in a humidified atmosphere at $37^{\circ} \mathrm{C}$ in $5 \% \mathrm{CO}_{2}$ and $95 \%$ air. In some experiments, the DRG neurons were pretreated overnight with pertussis toxin (PTX, $0.5 \mu \mathrm{g} / \mu \mathrm{l}$; List Biologic Laboratories, Campbell, CA).

L-Cell Transfection. L-cells (American Type Culture Collection, Manassas, VA) were cotransfected via electroporation with either ASIC1a or ASIC3 cDNA plasmids as described elsewhere (Farrag et al., 2017). Briefly, once cells reached $70 \%-80 \%$ confluency, they were incubated in trypsin for 3-5 minutes, and enzymatic activity was stopped with Dulbecco's modified Eagle's medium (supplemented with $10 \%$ FBS, $1 \%$ penicillin-streptomycin, $1 \%$ glutamine, and $10 \%$ nonessential amino acids).

The cell counts were determined with the Cell Countess (Thermo Fisher Scientific), and approximately 400,000 cells were transferred to the electroporation tip $(100 \mu \mathrm{l})$ of the NEON Electroporator (Thermo Fisher Scientific). The cells were electroporated with three 20-millisecond $1125 \mathrm{~V}$ pulses in a medium containing Opti-MEM (Thermo Fisher Scientific), 2,3-butanedione monoxime (2 mM), ASIC1a or ASIC3 constructs $(12 \mu \mathrm{g})$, and enhanced green fluorescent protein (EGFP, $3 \mu \mathrm{g}$ ) to facilitate identification of transfected L-cells. After electroporation, the cells were plated on $35-\mathrm{mm}$ dishes containing supplemented Dulbecco's modified Eagle's medium and returned to the cell incubator $\left(5 \% \mathrm{CO}_{2} / 95 \%\right.$ air $)$ at $37^{\circ} \mathrm{C}$.

Electrophysiology. After overnight incubation, electrophysiologic recordings of Dil-labeled DRG neurons or EGFP-expressing L-cells were performed with an Axopatch 200B amplifier (Molecular Devices, Sunnyvale, CA). ASIC currents were filtered at $2 \mathrm{kHz}$ with a four-pole low-pass Bessel filter, digitized at $10 \mathrm{kHz}$ with an ITC-18 A/D converter (HEKA Instruments, Holliston, MA), and were acquired with customdesigned F6 software written by Stephen R. Ikeda employing IGOR Pro (WaveMetrics, Lake Oswego, OR). The recording pipettes were made from 8250 glass (King Precision Glass, Claremont, CA), pulled on a P-97 puller (Sutter Instrument Co., Novato, CA), and coated with Sylgard (Dow Corning, Midland, MI). The holding potential $\left(\mathrm{V}_{\mathrm{H}}\right)$ for DRG neurons and L-cells was kept at -80 and $-60 \mathrm{mV}$, respectively.

ASIC currents were recorded at room temperature and activated by switching the external recording solutions, with varying $\mathrm{pH}$ and agents, with a gravity-fed perfusion system placed approximately $100 \mu \mathrm{m}$ from the cell. The complete solution exchange occurred within less than 1 second. Figure 1B shows the protocol employed to activate ASIC currents. Cells were bathed in control (pH 7.4) solution and thereafter exposed to a different control solution ( $\mathrm{pH}$ 6.0) for 10 seconds. This was repeated again, and the cells were then preincubated in control solution containing the test compound for 3 minutes, a period under which activation of ASIC channels was not observed. After the preincubation period, the cells were exposed to test solution ( $\mathrm{pH} 6.0$ ) + test compound for 10 seconds.

Recording Solutions. The internal pipette solution contained (in millimolar) $N$-methyl-D-glucamine 80 , tetraethylammonium hydroxide 20, $\mathrm{CsCl} 20, \mathrm{CsOH} 40$, Tris creatine phosphate 14, HEPES 10, $\mathrm{CaCl}_{2}$ 1, Mg-ATP 4, $\mathrm{Na}_{2}$ GTP 0.3, and EGTA 11 . The pH 7.2 was adjusted with $\mathrm{CH}_{3} \mathrm{SO}_{3}$, and the osmolality was $307 \mathrm{mOsm} / \mathrm{kg}$. The external (bath) solutions contained (in millimolar): $\mathrm{NaCl} 140, \mathrm{KCl} 5.4$, HEPES 10, $\mathrm{MgCl}_{2} 1, \mathrm{CaCl}_{2} 5$, glucose 15, $\mathrm{pH}$ 7.4. The external solution with a $\mathrm{pH} 6.0$ was buffered with $2-\mathrm{N}$-morpholino ethanesulfonic acid. The final $\mathrm{pH}$ was adjusted with $\mathrm{NaOH}$, and the osmolality ranged from 312 to $329 \mathrm{mOsm} / \mathrm{kg}$. Oxy, Fen, Rem (all from Sigma-Aldrich), and naloxone (Tocris Bioscience, Minneapolis, MN) stocks were prepared in water and were diluted in the external solutions to their final concentration.

Data Analysis and Research Design. The electrophysiologic recordings were both analyzed and generated with Igor Pro (WaveMetrics). The opioid-mediated potentiation of the sustained $\left(\mathrm{I}_{\mathrm{S}}\right)$ and peak $\left(\mathrm{I}_{\mathrm{P}}\right)$ ASIC currents were determined as described elsewhere (Farrag et al., 2017); they are illustrated in Fig. 1B in a cell during exposure to external $\mathrm{pH} 6.0$ alone or in the presence of $\mathrm{pH} 6.0+\mathrm{Oxy}$ $(10 \mu \mathrm{M})$. It should be noted that not all Dil-labeled cells that were tested expressed ASIC channels.

The figures shown were obtained with Autodesk Graphic software, and the statistical analysis was performed with Prism (GraphPad Software, La Jolla, CA) and expressed as mean \pm S.D. The sample sizes were prespecified before data collection. Data comparison between two groups was determined with the use of unpaired, twotailed Student's $t$ test, while one-way ANOVA followed by Tukey (Fig. 3) or Bonferroni (Fig. 4) post hoc tests were employed for multiple comparisons. $P<0.05$ was considered statistically significant. The intergroup comparisons were prespecified, and all results from the statistical tests are reported. The statistical comparisons performed with the groups shown in Fig. 3C (WKY vs. ASIC3 KO), Fig. 3D (WKY vs. ASIC3 KO vs. ASIC1 L-cells vs. ASIC3 L-cells), Fig. 4D (Oxy FP vs. Oxy LIG vs. Fen FP vs. Fen LIG vs. Rem FP vs. Rem LIG), and Fig. 4E (FP vs. LIG) were prespecified and not exploratory.

\section{Results}

Enhancement of Sustained ASIC Currents by Clinical Opioids in Rat DRG Neurons. In the first set of experiments, Dil-labeled neurons were exposed to opioids routinely employed for intraoperatively (Rem and Fen) or chronic pain (Oxy). Figure 2 shows superimposed ASIC current traces before (black traces) and during (red traces) application of Oxy (10 $\mu \mathrm{M}$, Fig. 2Ai), Fen (10 $\mu \mathrm{M}$, Fig. 2Bi), and Rem (10 $\mu \mathrm{M}$, Fig. 2Ci). It can be observed that the amplitude of the sustained currents increased after opioid exposure when compared with pH 6.0 alone.

The summary dot plot shown in Fig. 2D illustrates the increase in sustained ASIC current for all three opioids. The concentration $(10 \mu \mathrm{M})$ of clinical opioids chosen was based on our previous observations of the endomorphin pharmacologic profiles that showed maximal ASIC3 potentiation between 3 and $10 \mu \mathrm{M}$ (Farrag et al., 2017).

To determine whether the sustained current enhancement was mediated via stimulation of MOR, which couple to 


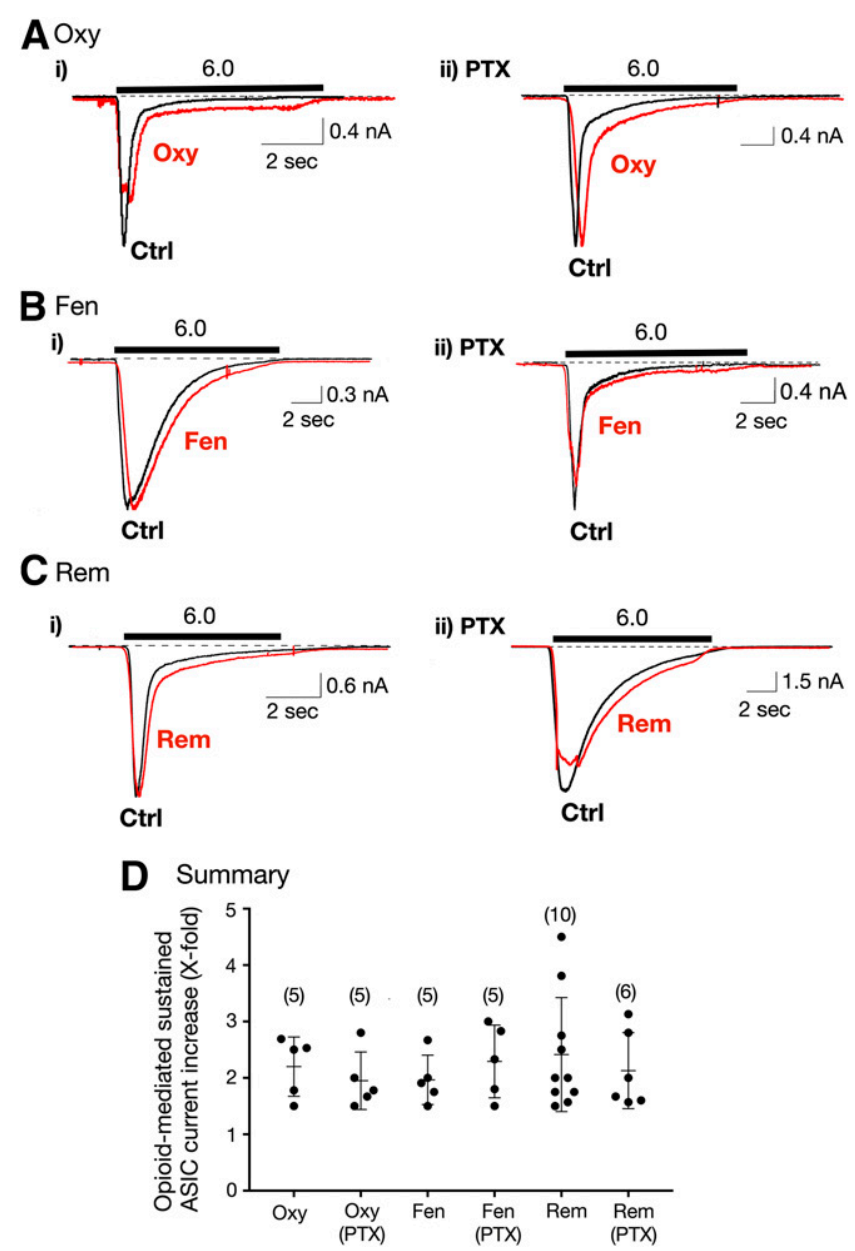

Fig. 2. Enhancement of sustained ASIC currents by opioids in Dil-labeled DRG neurons. (A-C) ASIC current traces from acutely isolated DRG neurons before (Ctrl [control], black trace) and after exposure to $10 \mu \mathrm{M}$ Oxy, Fen, and Rem in control (A-Ci) and in neurons pretreated overnight in PTX $(0.5 \mu \mathrm{g} / \mu \mathrm{l}$; A-Cii). The solid bars indicate the 10 -second exposure to either $\mathrm{pH} 6.0$ alone or $\mathrm{pH} 6.0+$ opioid. The cells were preexposed to the opioids ( $\mathrm{pH} 7.4$ ) for 3 minutes before application with $\mathrm{pH} 6.0+$ opioid. The holding potential $\left(\mathrm{V}_{\mathrm{H}}\right)$ for DRG neurons was $-80 \mathrm{mV}$. (D) Summary dot plot with mean ( \pm S.D.) of the Oxy-, Fen- and Rem-mediated enhancement of sustained ASIC currents in control and PTX-treated DRG neurons. The numbers in parenthesis indicate the number of recordings.

$\mathrm{G} \alpha_{\mathrm{i}} / \mathrm{G} \alpha_{\mathrm{o}}$ G proteins, a group of Dil-labeled neurons was pretreated overnight with PTX. The results in Fig. 2 (right) show that all three MOR agonists enhanced the sustained currents in a similar magnitude as that observed in control neurons. The results for all PTX-treated groups are summarized in Fig. 2D.

In a separate set of experiments, the MOR blocker naloxone was employed to determine whether Oxy would potentiate sustained currents in L-cells transfected with ASIC3 cDNA. The cells were first exposed to the external solution with $\mathrm{pH}$ 6.0, followed by $\mathrm{pH} 6.0+\mathrm{Oxy}(10 \mu \mathrm{M}$, see Materials and Methods above and protocol Fig. 1B). The cells were then pretreated with naloxone $(30 \mu \mathrm{M})$ and Oxy $(10 \mu \mathrm{M})$ in the control solution ( $\mathrm{pH}$ 7.4) for 3 minutes. Afterward, the cells were exposed to the solution ( $\mathrm{pH}$ 6.0) containing both naloxone $(30 \mu \mathrm{M})$ and Oxy $(10 \mu \mathrm{M})$. Under these conditions, ASIC3sustained currents were enhanced in external solutions containing either Oxy alone ( $\mathrm{pH}$ 6.0) or Oxy and naloxone (pH 6.0). The mean \pm S.D. (\%) Oxy-mediated ASIC3 current increases observed for Oxy alone and Oxy + naloxone were $83 \% \pm 68 \%$ and $472 \% \pm 386 \%(n=5, P=0.35)$, respectively. It should be noted that the higher increase observed with both Oxy + naloxone was a result of one cell exhibiting an enhancement of approximately 20 -fold. Overall, these results indicate that the opioids modulate the sustained currents independent of $\mathrm{G} \alpha_{\mathrm{i}} / \mathrm{G} \alpha_{0}$ subunits or MOR activation, which is consistent with our previous report (Farrag et al., 2017).

To examine whether the potentiation of sustained ASIC currents by opioids was a result of ASIC3 activation alone, we next isolated DRG neurons from control (WKY) and ASIC3 KO rats. The ASIC currents shown in Fig. 3A show that exposure to Rem $(10 \mu \mathrm{M})$ potentiated the sustained ASIC current almost 3 -fold. On the other hand, the application of Rem $(10 \mu \mathrm{M})$ to the neuron isolated from an ASIC3 $\mathrm{KO}$ rat failed to enhance the sustained ASIC currents (Fig. 3B). The summary dot plot in Fig. $3 \mathrm{C}$ indicates that Rem significantly $(P<0.001)$ enhanced ASIC currents when compared with DRG neurons isolated from ASIC3 KO rats. In 9 of 10 neurons, Rem exposure caused a slight inhibition of the sustained ASIC currents, and it nearly doubled the ASIC current in one neuron.

As mentioned previously, $\tau$ values for ASIC1 and ASIC3 are quite distinct. ASIC1 exhibit slow $\tau$, while $\tau$ for ASIC3 is fast (Kellenberger and Schild, 2015). Therefore, we next measured $\tau$ for both groups of DRG neurons. The plot in Fig. 3D indicates that $\tau$ values observed in WKY DRG neurons range from 0.1 to 4.4 seconds, indicative of ASIC current heterogeneity. However, the $\tau$ values of DRG neurons from ASIC3 KO were significantly $(P<0.001)$ greater when compared with control neurons. The lowest $\tau$ value for DRG neurons from KO rats was 1.6 seconds, which suggests that ASIC currents do not exhibit ASIC3-like $\tau$ values.

In a separate set of experiments, we measured $\tau$ values of L-cells transfected with either ASIC1a or ASIC3 cDNA. The $\tau$ values for L-cells expressing ASIC1a are similar in magnitude to neurons isolated from ASIC3 KO rats (Fig. 3D). The ASIC3expressing L-cells displayed $\tau$ values that were close to 0.3 seconds.

We next compared the effect of opioids on $\tau$ values in ASIC1a- and ASIC3-expressing L-cells. The $\tau$ values of the L-cell group expressing ASIC1a were $3.15 \pm 0.38$ and $2.47 \pm$ 0.70 seconds $(n=4 ; P=0.156$, paired $t$ test) before ( $\mathrm{pH} \mathrm{6.0)}$ and during ( $\mathrm{pH} 6.0+10 \mu \mathrm{M}$ Oxy) exposure, respectively. Additionally, the $\tau$ values for ASIC3-expressing L-cells were $0.37 \pm 0.08$ and $0.45 \pm 0.06$ seconds $(n=19 ; P=0.139$, paired $t$ test), before and during ( $\mathrm{pH} 6.0+10 \mu \mathrm{M}$ Oxy).

Effect of Muscle Ischemia on the Opioid-Mediated Enhancement of Sustained ASIC Currents in Rat DRG Neurons. Previous studies have shown that ASIC expression levels are altered under ischemic conditions such as that observed after femoral ligation (Liu et al., 2010; Xing et al., 2012). We have recently reported that ligation leads to both a decrease in ASIC1 and increase in ASIC3 expression levels in rat DRG neurons (Farrag et al., 2017). We thus compared the effect of the three clinical opioids on sustained ASIC currents in Dil-labeled neurons from both rats with freely perfused (FP, control) and ligated (LIG) femoral arteries.

The current traces shown in Fig. 4, $\mathrm{Ai}, \mathrm{Bi}$, and $\mathrm{Ci}$ are those obtained from control rats before (black traces) and during (red traces) opioid exposure. The traces shown to the right are those recorded in neurons isolated from rats with ligated arteries. The results summarized in Fig. 4D indicate that Oxy 
A WKY
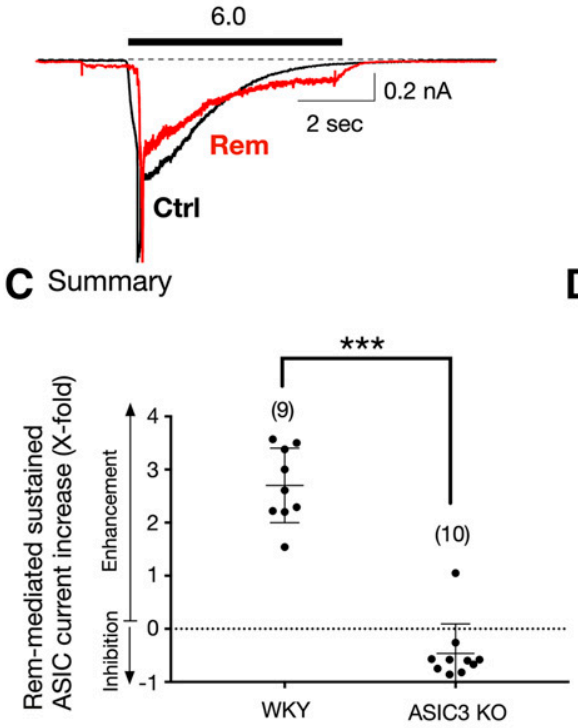

B ASIC3 KO

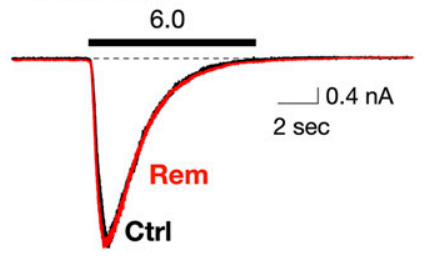

D Desensitization time constants $(\tau)$ $\star \star$

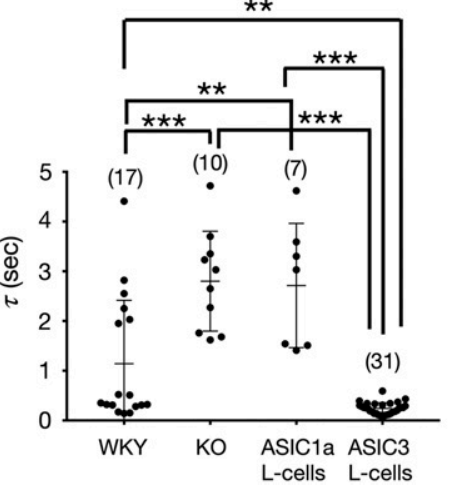

Fig. 3. Effect of Rem on sustained ASIC currents in acutely isolated Dil-labeled DRG neurons from WKY (control) and ASIC3 KO rats. (A and B) Representative ASIC current traces obtained from DRG neurons from WKY (A) and ASIC3 KO (B) rats before (Ctrl [control], black) and after Rem (10 $\mu \mathrm{M}$, red) exposure. The solid bars above the traces represent a 10 -second exposure to the $\mathrm{pH} 6.0$ test solution. The neurons were preexposed to Rem for 3 minutes $(\mathrm{pH}$ 7.4) before exposure to the test solutions (pH $6.0+$ Rem). (C) Summary dot plot indicating the mean ( \pm S.D.) Rem-mediated sustained ASIC current enhancement (X-fold). $* * * P<0.0001$ using unpaired two-tailed $t$ test. (D) Mean ( \pm S.D.) desensitization time constant $(\tau)$ values measured for ASIC currents in DRG neurons isolated from WKY rats (control), ASIC3 KO rats, and L-cells transfected with either ASIC1a or ASIC3 cDNA. The holding potential $\left(\mathrm{V}_{\mathrm{H}}\right)$ for DRG neurons and L-cells was -80 and $-60 \mathrm{mV}$, respectively. Numbers in parenthesis indicate the number of recordings. *** $P<0.0001$ and $* * P<0.001$, respectively, using one-way ANOVA followed by Tukey post hoc test. exposure led to a significantly $(P<0.05)$ greater enhancement of sustained ASIC currents. Although both Fen and Rem application exhibited greater sustained ASIC currents when compared with neurons from freely perfused muscle, no statistical significance was reached (Fig. 4D).

Figure $4 \mathrm{E}$ depicts the summary of the measured $\tau$ for both group of neurons. It can be observed that the mean $\tau$ of DRG neurons from ligated rats was significantly $(P<0.01)$ lower than that from control rats ( 2.41 vs. 0.88 seconds). The lower $\tau$, characteristic of ASIC3 channels, observed in neurons from the LIG rat group was likely a result of greater expression of this channel subunit. The mean $\tau$ for WKY KO rats (2.70 seconds; Fig. 3D) was comparable to that observed in Sprague-Dawley rats (Fig. 4E).

Effect of Opioids on Transient Peak Currents in Rat DRG Neurons and in ASIC-Transfected L-Cells. In the current study and previously (Farrag et al., 2017), we observed that whereas the opioids consistently enhanced the sustained ASIC currents, their effect on transient peak currents was mixed. That is, application of the opioids would increase, decrease, or have a minimal effect on the peak currents (see Figs. 2-4). Given that functional ASIC are trimeric, the actual ASIC1 and ASIC3 contribution to the total ASIC peak currents is difficult to ascertain. We reasoned that the varied responses may result from isoform heterogeneity in DRG neurons. Thus, we next used $\tau$ values to sort the DRG neurons isolated from FP and LIG rats into "ASIC1-like" and "ASIC3-like." That is, neurons with $\tau$ greater than 0.8 seconds were grouped into the former, and the rest were grouped into the latter.

Figure 5A is a summary dot plot of the change in transient peak currents after exposure to Oxy $(10 \mu \mathrm{M})$ in neurons grouped based on this criterion. The plot indicates that in ASIC1- and ASIC3-like neurons isolated from FP rats, Oxy application, for the most part, led to inhibition of the transient currents while in approximately $30 \%$ of cells in each group peak current enhancement was observed. We then compared the effect of Oxy on DRG neurons isolated from rats with ligated femoral arteries. In this group of neurons, Oxy application primarily blocked peak currents of ASIC1-like cells (6/7) but remained mixed in the ASIC3-like group of cells.

We next employed this criterion in L-cells expressing ASIC1 or ASIC3 alone (i.e., homomeric) or ASIC3 cotransfected with ASIC1a at a 1:1 and 1:3 ASIC3:ASIC1a ratio. The dot plot in Fig. 5B summarizes the effect of $\mathrm{Oxy}(10 \mu \mathrm{M})$ on the transient peak currents. The plot shows that Oxy exerted mixed effects on homomeric ASIC1 and ASIC3 peak currents. However, the coexpression of ASIC3 and ASIC1a led to mainly inhibitory effects with either a 1:1 or 1:3 ASIC3:ASIC1a ratio. This suggests that the opioid-mediated inhibitory of peak currents are more likely to occur under conditions where both ASIC1a and ASIC3 isoforms are coexpressed.

We previously found that E-2 caused significant potentiation of sustained ASIC currents. Thus, in the next set of experiments we examined whether coapplication of E-2 and Oxy would exert primarily potentiating effects on ASIC peak currents. DRG neuron from LIG rats, with presumably greater ASIC3 expression levels, and L-cells transfected with ASIC1a and/or ASIC3 were preincubated in Oxy ( $\mathrm{pH} 7.4)$ for 3 minutes. Thereafter, both E-2 and Oxy (both at $10 \mu \mathrm{M}$ ) were applied to the cells. The results in Fig. 5A indicate that in ASIC1-like cells, E-2 + Oxy primarily (6/8) blocked peak currents but enhanced the currents in most of ASIC3-like cells (8/10). Similarly, in L-cells coexpressing both ASIC channel subunits resulted in block of the peak currents by both agonists. Yet both E-2 and Oxy potentiated all $(n=9)$ ASIC3-expressing L-cells, though in one cell the enhancement was relatively small.

\section{Discussion}

The results of our present study demonstrate that the synthetic and semisynthetic opioids Oxy, Fen, and Rem, like endogenous opioid peptides, can potentiate sustained ASIC currents in acutely isolated DRG neurons innervating triceps surae muscle. The opioid-mediated potentiation was also significantly greater in DRG neurons isolated from rats with ligated femoral arteries, a model of PAD. Our findings further suggest 
A Oxy

i) $\mathrm{FP}$

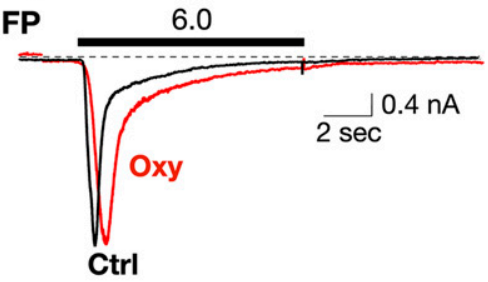

B Fen

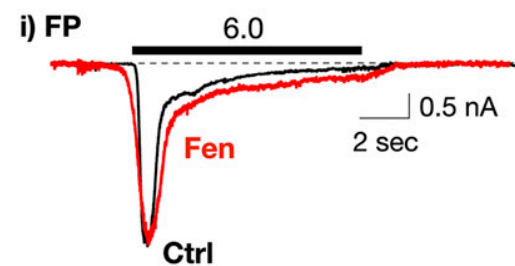

C Rem
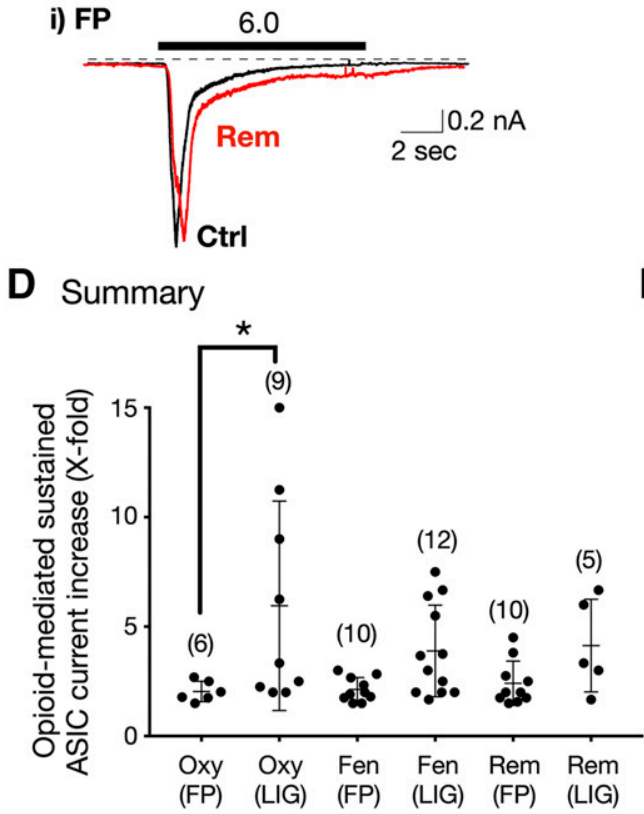

ii) LIG

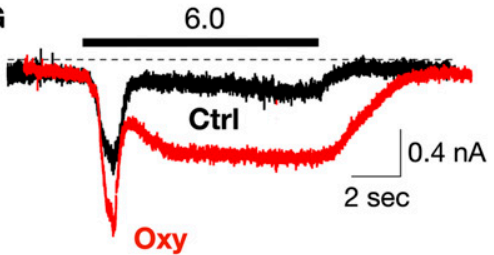

ii) LIG

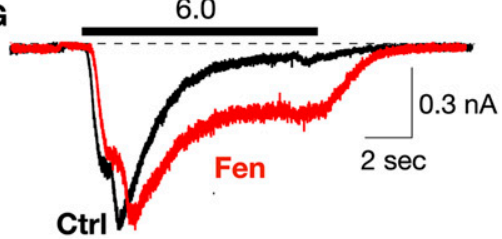

ii) LIG

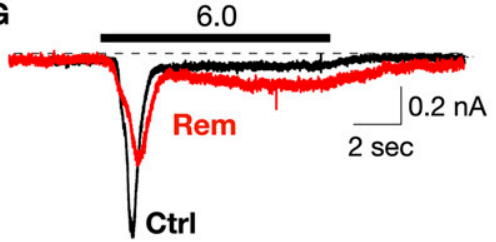

E Desensitization time constants $(\tau)$

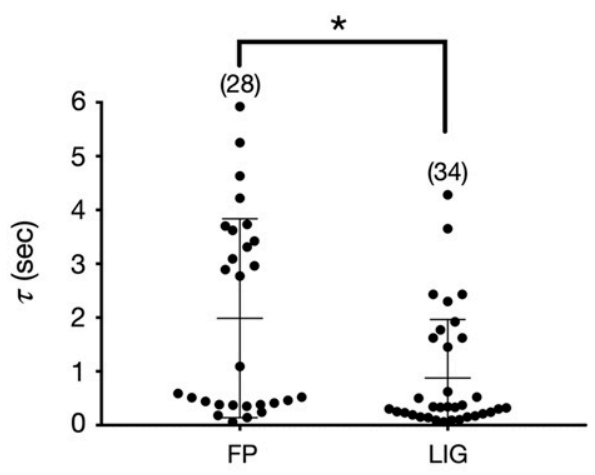

Fig. 4. Enhancement of sustained ASIC currents by opioids in Dil-labeled DRG neurons isolated from freely perfused (FP) and femoral-ligated (LIG) rats. (A-C) ASIC current traces from FP and LIG rat DRG neurons before (Ctrl [control], black) and after Oxy (Ai), Fen (Bi), and Rem (Ci, red) exposure. The solid bars above the traces represent a 10-second exposure to the $\mathrm{pH}$ 6.0 test solution. The neurons were preexposed to opioids for 3 minutes ( $\mathrm{pH} 7.4$ ) just before exposure to the test solutions (pH 6.0). The holding potential $\left(\mathrm{V}_{\mathrm{H}}\right)$ was $-80 \mathrm{mV}$. (D) Summary dot plot with mean ( \pm S.D.) of opioid-mediated sustained ASIC currents (X-fold) potentiation from $\mathrm{FP}$ and LIG rats. $* P<0.01$ using one-way ANOVA followed by Bonferroni multiple comparisons test. (E) Mean ( \pm S.D.) desensitization time constant $(\tau)$ values measured for ASIC currents obtained from FP and LIG DRG neurons. ${ }^{*} P<0.01$ using unpaired two-tailed $t$ test. Numbers in parentheses indicate the number of recordings. that the enhancement of the sustained currents does not result from stimulation of $\mathrm{G}$ protein subunits $\left(\mathrm{G} \alpha_{\mathrm{i}} / \mathrm{G} \alpha_{\mathrm{o}}\right)$, known to couple to MOR in rat DRG neurons (Hassan et al., 2014), because PTX pretreatment did not prevent current potentiation. Additionally, enhancement of the sustained ASIC3 currents occurred in the presence of the MOR blocker naloxone, suggesting that MOR stimulation is not involved. Rather, it is likely that the opioids interact directly with ASIC3 channel subunits. Moreover, the three opioids exerted varying effects on the peak transient currents: enhanced, blocked, or no effect. Thus, the overall modulation of ASIC currents by opioids appears to be multifactorial.

Most studies that have reported the effect of inflammatory mediators on ASIC currents have focused on peak transient currents. Dynorphins, for example, are thought to interact with ASIC1 channel subunits that potentiate ASIC currents (Sherwood et al., 2012). Similar observations have been reported with FFRM amides (Askwith et al., 2000). In cases where it is shown that there is crosstalk between the receptor and ASIC channels, this has also reported the effect on peak transient currents. Unlike these studies, however, our results show that the clinical opioids can exert variable effects, as has also been observed with endogenous opioid peptides (Farrag et al., 2017).

In the present study, we employed the desensitization time constant as an indicator of ASIC isoform influence on wholecell ASIC current response to opioids. Sorting $\tau$ values into "ASIC1"- and "ASIC3"-like showed that exposure of DRG neurons to opioids will exert primarily an inhibitory effect on transient peak currents. Under conditions where ASIC3 expression dominates (i.e., DRG from LIG group), a greater fraction of peak currents will be potentiated by opioids. The varied responses demonstrate that ASIC stoichiometry exhibits a complex pharmacologic profile resulting from the sum of two independent types of actions that comprise whole-cell ASIC currents. These observations, which have been previously reported by others (Babinski et al., 2000; Hesselager et al., 2004; Hattori et al., 2009; Kusama et al., 2013), are thought to serve as a regulatory mechanism in response to slight changes in extracellular $\mathrm{pH}$. 


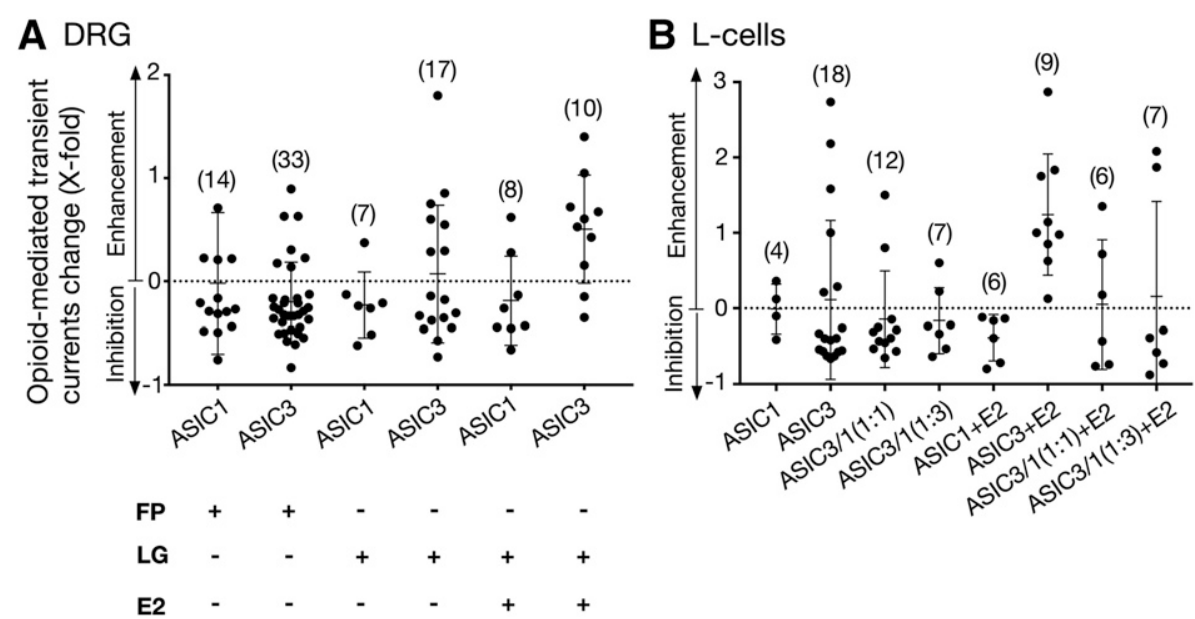

\begin{abstract}
Fig. 5. Effect of Oxy on ASIC peak transient currents in acutely isolated Dil-labeled DRG neurons and L-cells transfected with ASIC3 alone or with ASIC1a. (A) Summary dot plot with mean ( \pm S.D.) percentage change of Oxy-mediated modulation on transient currents after application of $\mathrm{Oxy}(10 \mu \mathrm{M})$ alone or $\mathrm{Oxy}+\mathrm{E}-2(10 \mu \mathrm{M})$ in DRG neurons from FP and LIG rats (A) and L-cells (B) transfected with ASIC3 or ASIC3 with ASIC1a. The cells were first preincubated with Oxy for 3 minutes ( $\mathrm{pH} 7.4$ ), and this was followed by a 10-second application of Oxy alone or Oxy + E-2 ( $\mathrm{pH}$ 6.0). The holding potential $\left(V_{\mathrm{H}}\right)$ for DRG neurons and L-cells was -80 and $-60 \mathrm{mV}$, respectively. Numbers in parentheses indicate the number of cells tested.
\end{abstract}

One study that examined the effect of either morphine or $\left[\mathrm{D}-\mathrm{Ala}^{2}, \mathrm{~N}-\mathrm{MePhe}^{4}, \mathrm{Gly}-\mathrm{ol}\right]-$ enkephalin (DAMGO) in rat DRG found that either agonist blocked the peak transient currents (Cai et al., 2014). However, the effect on sustained ASIC currents was not reported. Unlike a previous report (Farrag et al., 2017) and our present study, the investigators showed that the inhibition of the peak transient currents was due to crosstalk between MOR and ASIC isoforms that employed cAMP signaling events. Although morphine and Oxy share similar structures, we observed sustained ASIC current enhancement in the presence of PTX and in L-cells that do not express MOR. Our results also show that Oxy exerted variable effects on peak currents particularly in DRG neurons from FP rats, though most were inhibitory.

Ischemic pain, often observed with claudication and angina, is associated with severe muscle ischemia. The sustained component of ASIC currents is thought to play a major role in pain and pressor responses associated with tissue ischemia as well as inflammation (Naves and McCleskey, 2005; Salinas et al., 2009; Sluka and Gregory, 2015). The sustained current appears to be responsible for the long-lasting pain observed under chronic ischemic conditions. Previous studies have reported that ASIC isoform expression levels in sensory neurons are altered under inflammatory or ischemic conditions. For instance, the carrageenan-induced muscle inflammation in mice has been reported to increase ASIC2 and ASIC3 expression levels while ASIC1 is unchanged (Walder et al., 2010). Femoral ligation in rats has been shown to significantly increase ASIC3 expression while decreasing ASIC1 levels (Liu et al., 2010; Xing et al., 2012; Farrag et al., 2017).

In our current study, the synthetic opioids, particularly Oxy, significantly enhanced the sustained ASIC currents in DRG neurons from rats with ligated femoral arteries. This group of neurons also exhibited a significantly lower desensitization constant in the same group of neurons, which is indicative of greater ASIC3 contribution to the total ASIC currents. In addition, the effect of Oxy on ASIC3 channel currents increased $\tau$ values by approximately $20 \%$, while $\tau$ for ASIC 1 currents decreased 20\%. These slight changes suggest Oxy exposure may increase the response of ASIC3 channels to acid under acidic conditions, whereas the opposite would occur with ASIC1. More recently, E-1 was shown to also slow desensitization of ASIC3 currents recorded in Xenopus oocytes (Vyvers et al., 2018).
Although the amplitude of native sustained ASIC currents is relatively smaller than the peak current, it persists as long as the extracellular $\mathrm{pH}$ remains acidic. The sustained ASIC3 currents have been shown to made up of the overlap of both activation and inactivation curves, also referred to as window currents (Yagi et al., 2006). Signaling molecules, such as arachidonic acid, lysophosphatidylcholine, and endomorphins, enhance the window currents by both shifting the $\mathrm{pH}$ activation curve leftward to more basic $\mathrm{pH}$ values and the inactivation curve rightward to more acidic values (Deval et al., 2008; Marra et al., 2016; Farrag et al., 2017). The end result is an increase in the probability that closed noninactivated ASIC3 channels will open within this $\mathrm{pH}$ range when exposed these signaling elements and increase nociceptor excitability.

Our observations of the opioid-mediated sustained ASIC current potentiation bear physiologic relevance to the pathologic conditions such as PAD. Figure 6 illustrates our hypothesis by which OIH can occur from muscle ischemia and long-term opioid use. Under conditions where exercising muscle is freely perfused (Fig. 6A), lactic acid release will decrease extracellular $\mathrm{pH}$. As a result, $\mathrm{E}-2$ is released by DRG neurons in the muscle and dorsal horn.

Note that $\mathrm{Ca}^{2+}$ ions are chelated by lactic acid, which potentiates ASIC activity. At the presynapse, the afferentmediated release of E-2 causes MOR stimulation such that the ascending pain signaling pathway is blocked. Figure $6 \mathrm{~B}$ depicts an ischemic muscle with low blood flow that is accompanied by the following: 1) an increase in local inflammatory mediators, 2) an increase in ASIC3 isoform expression, 3) a decrease in ASIC1 expression, 4) an increase in E-2 release, and 5) an increase in $\mathrm{Ca}^{2+}$ ion chelation by lactic acid. The end result is sensory neuron hyperexcitability and pain.

Claudication pain can be alleviated with prescription opioids such as Oxy (Samolsky Dekel et al., 2010). However, the pain relief comes at the cost of developing opioid tolerance and eventual addiction. In some cases, it is likely that opioid tolerance can also lead to OIH. That is, with the continued use of opioid medication and possibly the increased E-2 release at the ischemic site, patients exhibit sensitization to painful stimuli. This has been documented with Rem and Fen (Lee et al., 2011; Kim et al., 2014; Santonocito et al., 2018).

In summary, our results show that three clinically employed, high-affinity MOR agonists potentiate ASIC currents in DRG neurons, with a more pronounced effect in neurons isolated 


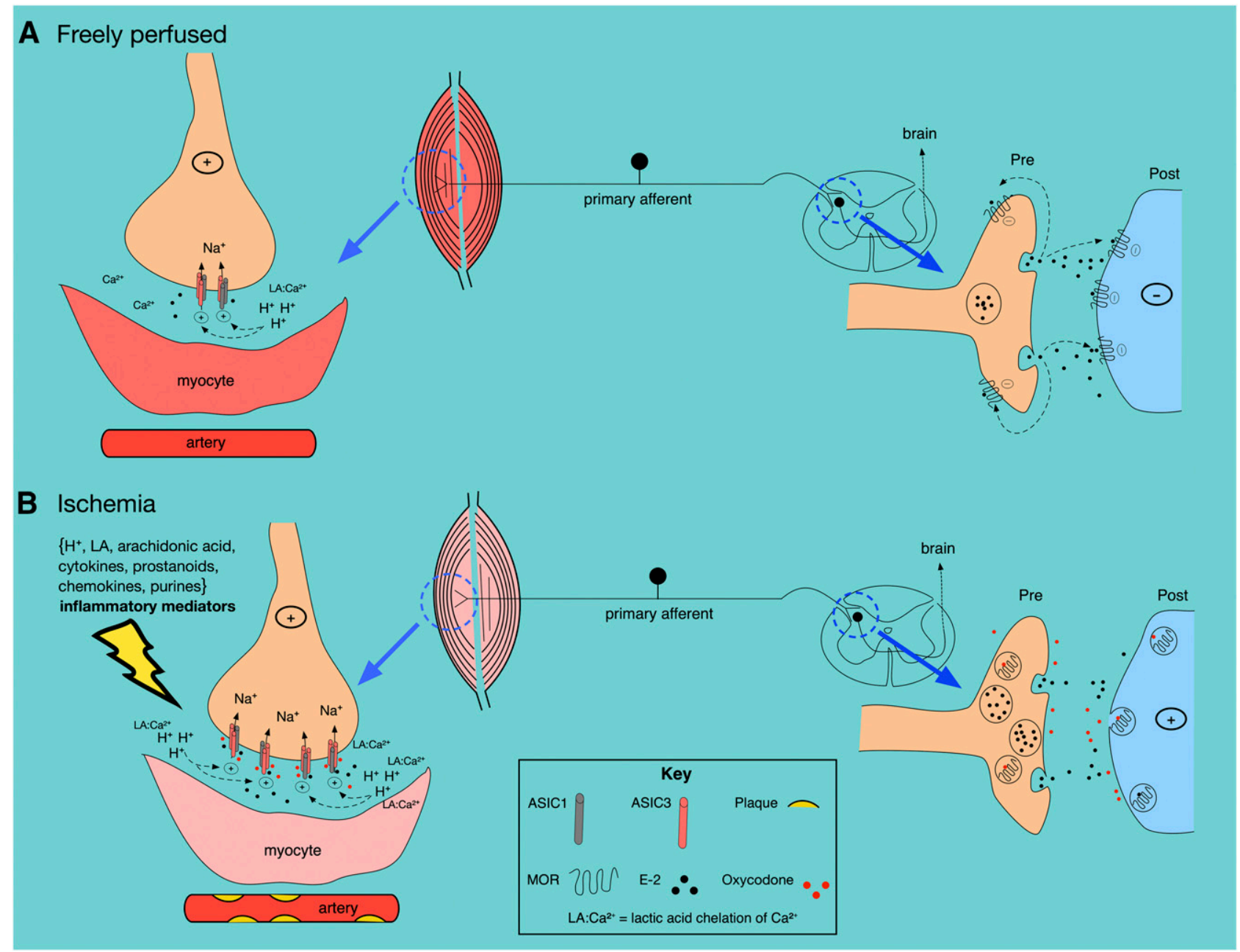

Fig. 6. General scheme showing possible opioid- and endomorphin-mediated effects on ASIC channels under freely perfused (A) and ischemic (B) conditions. See the text for details.

from animals with ligated arteries. Like other inflammatory mediators, such as lactic acid, arachidonic acid, dynorphin peptides, and endomorphins, these opioids can effectively modulate ASIC currents independent of receptor activation. The results identify a novel, direct interaction of ASIC3 channel isoforms with opioids. This interaction may help to explain and understand the molecular mechanism associated with OIH that is occurs without MOR stimulation.

\section{Acknowledgments}

We gratefully acknowledge Paul B. Herold (Department of Anesthesiology and Perioperative Medicine, Penn State College of Medicine) for his technical assistance and Dr. M. Dwinell and Dr. A. Geurts and Genome Editing Rat Resource Center (HL-114474) for generation of the ASIC3 KO rats.

\section{Authorship Contributions}

Participated in research design: Zaremba, Ruiz-Velasco.

Conducted experiments: Zaremba, Ruiz-Velasco.

Performed data analysis: Zaremba, Ruiz-Velasco.

Wrote or contributed to writing of the manuscript: Zaremba, RuizVelasco.

\section{References}

Askwith CC, Cheng C, Ikuma M, Benson C, Price MP, and Welsh MJ (2000) Neuropeptide FF and FMRFamide potentiate acid-evoked currents from sensory neurons and proton-gated DEG/ENaC channels. Neuron 26:133-141.
Babinski K, Catarsi S, Biagini G, and Séguéla P (2000) Mammalian ASIC2a and ASIC3 subunits co-assemble into heteromeric proton-gated channels sensitive to Gd3+. J Biol Chem 275:28519-28525.

Baron A and Lingueglia E (2015) Pharmacology of acid-sensing ion channels physiological and therapeutical perspectives. Neuropharmacology 94:19-35.

Cai Q, Qiu CY, Qiu F, Liu TT, Qu ZW, Liu YM, and Hu WP (2014) Morphine inhibits acid-sensing ion channel currents in rat dorsal root ganglion neurons. Brain Res 1554:12-20.

Copp SW, Kim JS, Ruiz-Velasco V, and Kaufman MP (2016) The mechano-gated channel inhibitor GsMTx4 reduces the exercise pressor reflex in rats with ligated femoral arteries. Am J Physiol Heart Circ Physiol 310:H1233-H1241.

Criqui MH and Aboyans V (2015) Epidemiology of peripheral artery disease. Circ Res 116:1509-1526.

Deval E, Noël J, Lay N, Alloui A, Diochot S, Friend V, Jodar M, Lazdunski M, and Lingueglia E (2008) ASIC3, a sensor of acidic and primary inflammatory pain. EMBO J 27:3047-3055.

Deval E and Lingueglia E (2015) Acid-sensing ion channels and nociception in the peripheral and central nervous systems. Neuropharmacology 94:49-57.

Farrag M, Drobish JK, Puhl HL, Kim JS, Herold PB, Kaufman MP, and Ruiz-Velasco V (2017) Endomorphins potentiate acid-sensing ion channel currents and enhance the lactic acid-mediated increase in arterial blood pressure: effects amplified in hindlimb ischaemia. $J$ Physiol 595:7167-7183.

Fletcher D and Martinez V (2014) Opioid-induced hyperalgesia in patients after surgery: a systematic review and a meta-analysis. Br J Anaesth 112:991-1004.

Gründer S and Pusch M (2015) Biophysical properties of acid-sensing ion channels (ASICs). Neuropharmacology 94:9-18.

Hassan B, Kim JS, Farrag M, Kaufman MP, and Ruiz-Velasco V (2014) Alteration of the mu opioid receptor: $\mathrm{Ca} 2+$ channel signaling pathway in a subset of rat sensory neurons following chronic femoral artery occlusion. J Neurophysiol 112: $3104-3115$.

Hattori T, Chen J, Harding AM, Price MP, Lu Y, Abboud FM, and Benson CJ (2009) ASIC2a and ASIC3 heteromultimerize to form pH-sensitive channels in mouse cardiac dorsal root ganglia neurons. Circ Res 105:279-286.

Hesselager M, Timmermann DB, and Ahring PK (2004) pH Dependency and desensitization kinetics of heterologously expressed combinations of acid-sensing ion channel subunits. J Biol Chem 279:11006-11015. 
Immke DC and McCleskey EW (2001) Lactate enhances the acid-sensing $\mathrm{Na}+$ channel on ischemia-sensing neurons. Nat Neurosci 4:869-870.

Kellenberger S and Schild L (2015) International Union of Basic and Clinical Pharmacology. XCI. Structure, function, and pharmacology of acid-sensing ion channels and the epithelial $\mathrm{Na}^{+}$channel. Pharmacol Rev 67:1-35.

Kim SH, Stoicea N, Soghomonyan S, and Bergese SD (2014) Intraoperative use of remifentanil and opioid induced hyperalgesia/acute opioid tolerance: systematic review. Front Pharmacol 5:108.

Kusama N, Gautam M, Harding AMS, Snyder PM, and Benson CJ (2013) Acidsensing ion channels (ASICs) are differentially modulated by anions dependent on their subunit composition. Am J Physiol Cell Physiol 304:C89-C101.

Lash JM, Nixon JC, and Unthank JL (1995) Exercise training effects on collateral and microvascular resistances in rat model of arterial insufficiency. Am J Physiol 268:H125-H137.

Lee M, Silverman SM, Hansen H, Patel VB, and Manchikanti L (2011) A comprehensive review of opioid-induced hyperalgesia. Pain Physician 14:145-161.

Liu J, Gao Z, and Li J (2010) Femoral artery occlusion increases expression of ASIC3 in dorsal root ganglion neurons. Am $J$ Physiol Heart Circ Physiol 299: H1357-H1364.

Marra S, Ferru-Clément R, Breuil V, Delaunay A, Christin M, Friend V, Sebille S, Cognard C, Ferreira T, Roux C, et al. (2016) Non-acidic activation of pain-related acid-sensing ion channel 3 by lipids. EMBO J 35:414-428.

Molliver DC, Immke DC, Fierro L, Paré M, Rice FL, and McCleskey EW (2005) ASIC3, an acid-sensing ion channel, is expressed in metaboreceptive sensory neurons. Mol Pain 1:35.

Mousa SA, Machelska H, Schäfer M, and Stein C (2002) Immunohistochemical localization of endomorphin-1 and endomorphin-2 in immune cells and spinal cord in a model of inflammatory pain. $J$ Neuroimmunol 126:5-15.

Naves LA and McCleskey EW (2005) An acid-sensing ion channel that detects ischemic pain. Braz J Med Biol Res 38:1561-1569.

Salinas M, Lazdunski M, and Lingueglia E (2009) Structural elements for the generation of sustained currents by the acid pain sensor ASIC3. J Biol Chem 284 31851-31859.
Samolsky Dekel BG, Melotti RM, Gargiulo M, Freyrie A, Stella A, and Di Nino G (2010) Pain management in peripheral arterial obstructive disease: oral slow-release oxycodone versus epidural L-bupivacaine. Eur J Vasc Endovasc Surg 39:774-778.

Santonocito C, Noto A, Crimi C, and Sanfilippo F (2018) Remifentanil-induced postoperative hyperalgesia: current perspectives on mechanisms and therapeutic strategies. Local Reg Anesth 11:15-23.

Sherwood TW and Askwith CC (2009) Dynorphin opioid peptides enhance acidsensing ion channel 1a activity and acidosis-induced neuronal death. $J$ Neurosci 29:14371-14380.

Sherwood TW, Frey EN, and Askwith CC (2012) Structure and activity of the acidsensing ion channels. Am J Physiol Cell Physiol 303:C699-C710.

Sluka KA and Gregory NS (2015) The dichotomized role for acid sensing ion channels in musculoskeletal pain and inflammation. Neuropharmacology 94:58-63.

Smith ES, Cadiou H, and McNaughton PA (2007) Arachidonic acid potentiates acidsensing ion channels in rat sensory neurons by a direct action. Neuroscience $\mathbf{1 4 5}$ $686-698$.

Vyvers A, Schmidt A, Wiemuth D, and Gründer S (2018) Screening of 109 neuropeptides on ASICs reveals no direct agonists and dynorphin A, YFMRFamide and endomorphin-1 as modulators. Sci Rep 8:18000.

Walder RY, Rasmussen LA, Rainier JD, Light AR, Wemmie JA, and Sluka KA (2010) ASIC1 and ASIC3 play different roles in the development of hyperalgesia after inflammatory muscle injury. J Pain 11:210-218.

Xing J, Lu J, and Li J (2012) Acid-sensing ion channel subtype 3 function and immunolabelling increases in skeletal muscle sensory neurons following femora artery occlusion. J Physiol 590:1261-1272.

Yagi J, Wenk HN, Naves LA, and McCleskey EW (2006) Sustained currents through ASIC3 ion channels at the modest $\mathrm{pH}$ changes that occur during myocardial ischemia. Circ Res 99:501-509.

Address correspondence to: Dr. Victor Ruiz-Velasco, Department of Anesthesiology and Perioperative Medicine, Penn State College of Medicine, 500 University Drive, Hershey, PA 17033-0850. E-mail: vjr10@psu.edu 